\title{
Efficacy and Safety of Transurethral Laser Surgery Versus Transurethral Resection for Non-Muscle-Invasive Bladder Cancer: A Meta-Analysis and Systematic Review
}

\author{
Jiangnan Xu Chao Wang Jun Ouyang Jiale Sun Can Hu
}

Department of Urology, The First Affiliated Hospital of Soochow University, Suzhou, China

\section{Keywords}

Bladder cancer · Laser surgery · Transurethral resection ·

Systematic review and meta-analysis

\begin{abstract}
Objective: To compare the efficacy and safety of transurethral laser surgery and transurethral resection of a bladder tumor (TURBT) for non-muscle-invasive bladder cancer (NMIBC). Material and Methods: A research was carried out in Medline via PubMed, EMBASE, the Cochrane Library, and Web of Science up to October 20, 2019, to identify articles related to transurethral laser surgery and TURBT for NMIBC. All analyses were done using RevMan5.3 and Stata14. Results: A total of 17 studies involving 2,439 participants were included. The analysis showed no significant difference in operation times (mean difference $=-0.2 ; 95 \% \mathrm{Cl}-2.29$ to $1.89 ; p=0.85$ ) or occurrences of urethral stricture $(\mathrm{OR}=0.7 ; 95 \% \mathrm{Cl} 0.24-2.06 ; p=$ $0.52)$. Transurethral laser surgery was associated with a lower incidence of obturator nerve reflex $(\mathrm{OR}=0.04 ; 95 \% \mathrm{Cl} 0.02-$ $0.09 ; p<0.00001)$ and bladder perforation $(\mathrm{OR}=0.09 ; 95 \% \mathrm{Cl}$ $0.04-0.23 ; p<0.00001)$, a higher rate of detrusor muscle acquisition (OR $=5.28 ; 95 \% \mathrm{Cl} 2.42-11.49 ; p<0.0001)$, shorter catheterization (mean difference $=-1.05 ; 95 \% \mathrm{Cl}-1.41$ to $-0.68 ; p<0.00001$ ) and hospitalization times (mean difference $=-0.96 ; 95 \% \mathrm{Cl}-1.59$ to $-0.33 ; p=0.003$ ), and lower rates
\end{abstract}

karger@karger.com

(c) 2020 S. Karger AG, Basel

www.karger.com/uin

Karger! of bladder irrigation ( $\mathrm{OR}=0.21 ; 95 \% \mathrm{Cl} 0.13-0.35 ; p<0.00001$ ) and recurrence both at 12 months $(\mathrm{OR}=0.66 ; 95 \% \mathrm{Cl} 0.48-0.9$, $p=0.008)$ and at 24 months (OR $=0.6 ; 95 \% \mathrm{Cl} 0.41-0.86 ; p=$ $0.005)$. Conclusions: Transurethral laser surgery for NMIBC, as compared to TURBT, is associated with a lower incidence of complications, a lower recurrence rate, and faster postoperative recovery.

(c) 2020 S. Karger AG, Basel

\section{Introduction}

Bladder cancer is one of the most common malignant tumors of the urinary system. The American Cancer Society estimates that there will be 80,470 new cases of bladder cancer and 17,670 cancer-related deaths in 2019 [1]. More than $95 \%$ of bladder cancers are urothelial carcinomas, and non-muscle-invasive bladder cancer (NMIBC) accounts for $75-80 \%$ of urothelial carcinomas, including bladder tumors confined to the mucosa (stage Ta, carcinoma in situ) or submucosa (stage T1) [2].

Currently, the standard treatment for NMIBC is transurethral resection of bladder tumor (TURBT) followed by

Jiangnan Xu and Chao Wang contributed equally to this paper. 
adjuvant intravesical chemotherapy or immunotherapy $[3,4]$. Although TURBT is very mature, it is still difficult to control the cutting depth when the tumor is close to the ureteral orifice; the obturator nerve reflex may occur when the tumor is located in the lateral wall, leading to bladder perforation and even iliac vascular injury $[5,6]$. At the same time, piecemeal resection as an "incise and scatter" process is associated with an increased risk of tumor recurrence and difficulties accurately assessing pathological staging of tumors due to incomplete resection [7-9].

With the development of laser technology, the first transurethral NMIBC laser surgery occurred in the 1970 s [10]. It was approved by the FDA in 1984 and has been widely used in recent years due to its good tissue vaporization and hemostatic effect as well as its high safety [10, 11]. In particular, the use of laser surgery for en bloc resection can provide an intact tissue specimen for accurate pathological evaluation and reduce the risk of scattering malignant cells [12]. Several kind of lasers have been found to be useful in transurethral laser therapy for NMIBC, with holmium and 2-um lasers being the most commonly used in clinical practice [13]. With the increasingly extensive application of laser surgery, whether laser surgical treatment NMIBC can replace TURBT as the gold standard for NMIBC or only as a supplement to TURBT has become a hot issue. In recent years, several studies have directly compared transurethral laser therapy with TURBT in an attempt to explore this issue and they have yielded similar, but not completely consistent, results on some oncology and perioperative indicators [14-16]. At the same time, these existing studies all have shortcomings such as a small sample size, being single center, and a lack of randomization, and some of them are only observational studies so the results obtained may exaggerate or reduce the real difference in efficacy and safety between transurethral laser surgery and TURBT. This paper shows a meta-analysis of the previous literature in order to more accurately compare the efficacy and safety of transurethral laser surgery and TURBT treatment of NMIBC and to explore whether transurethral laser surgery can replace TURBT so as to provide a reference for the selection of clinical treatment options.

\section{Methods}

Search Strategy

A systematic search was carried out in Medline via PubMed, EMBASE, the Cochrane Library, and Web of Science up to October 20,2019, to identify published articles related to transurethral laser surgery and TURBT for NMIBC. The types of studies included randomized controlled trials (RCT), cohort studies, and clinical case-control studies. The search terms used included: (bladder cancer OR bladder tumor OR bladder carcinoma OR noninvasive bladder cancer OR urothelial carcinoma) and (transurethral resection OR resection) and (laser). We also browsed the list of references for highly relevant literature and manually searched the grey literature to ensure that no relevant literature was missing. This system review and meta-analysis is reported in accordance with the preferred reporting items of the system review and meta-analysis (PRISMA) [17] (ISSM_PRISMA_Checklist.pdf; for all online suppl. material, see www.karger.com/doi/10.1159/000506655).

\section{Inclusion and Exclusion Criteria}

The inclusion criteria were: (1) the types of studies were RCT or cohort studies and clinical case-control studies; (2) the study subjects were primary NMIBC patients with a clear pathological diagnosis and postoperative bladder perfusion therapy, followed up for more than 1 year; (3) interventions included all laser types of transurethral laser surgery; (4) studies involved the comparison of transurethral laser surgery and TURBT; and (5) outcome indicators included at least one of the following: operation time, incidence of obturator nerve reflex, incidence of bladder perforation, detrusor muscle acquisition rate, hospitalization time, catheterization time, bladder irrigation rate, incidence of urethral stricture, and recurrence rate.

The exclusion criteria were: (a) the subjects included patients with recurrent or muscle-invasive bladder cancer; (b) ta lack of relevant outcome indicators; (c) the patient had undergone other transurethral procedures; and (d) unclear outcomes and missing data; and (e) republished literature.

\section{Selection Process and Data Abstraction}

Two reviewers reviewed the titles, abstracts, and full text independently according to the inclusion and exclusion criteria. Disputes arising during the title and abstract screening phase were directly incorporated into the full text assessment to ensure that all relevant papers were not omitted. In the full text stage, the differences were resolved by negotiation between 2 reviewers. If there was no agreement, a third reviewer is consulted.

The 2 reviewers independently extracted relevant data with a predesigned data extraction table. Baseline data extracted included: first author and publication year, country, study type, surgical methods, sample size, follow-up time, outcomes, and quality score. Outcome indexes can be divided into intraoperative indexes and postoperative indexes. Intraoperative indicators include operation time, incidence of obturator nerve reflex, incidence of perforation, and detrusor muscle acquisition rate. Postoperative outcome indicators included: hospitalization time, catheterization time, bladder irrigation rate, incidence of urethral stricture, 12-month recurrence rate, and 24-month recurrence rate. The median, range, and sample size data provided in the literature were all converted into the data types required for the statistics in this study by the method described by Hozo et al. [18].

\section{Literature Quality and Risk of Bias Assessment}

To assess the quality of the literature and the risk of bias, we used the Jadad score [19] to evaluate RCT and the Newcastle-Ottawa Scale (NOS) [20] to evaluate retrospective cohort studies. The Jadad score mainly evaluates randomization and randomization
Transurethral Laser Surgery vs.

Transurethral Resection for NMIBC
Urol Int 2020;104:810-823

DOI: $10.1159 / 000506655$ 


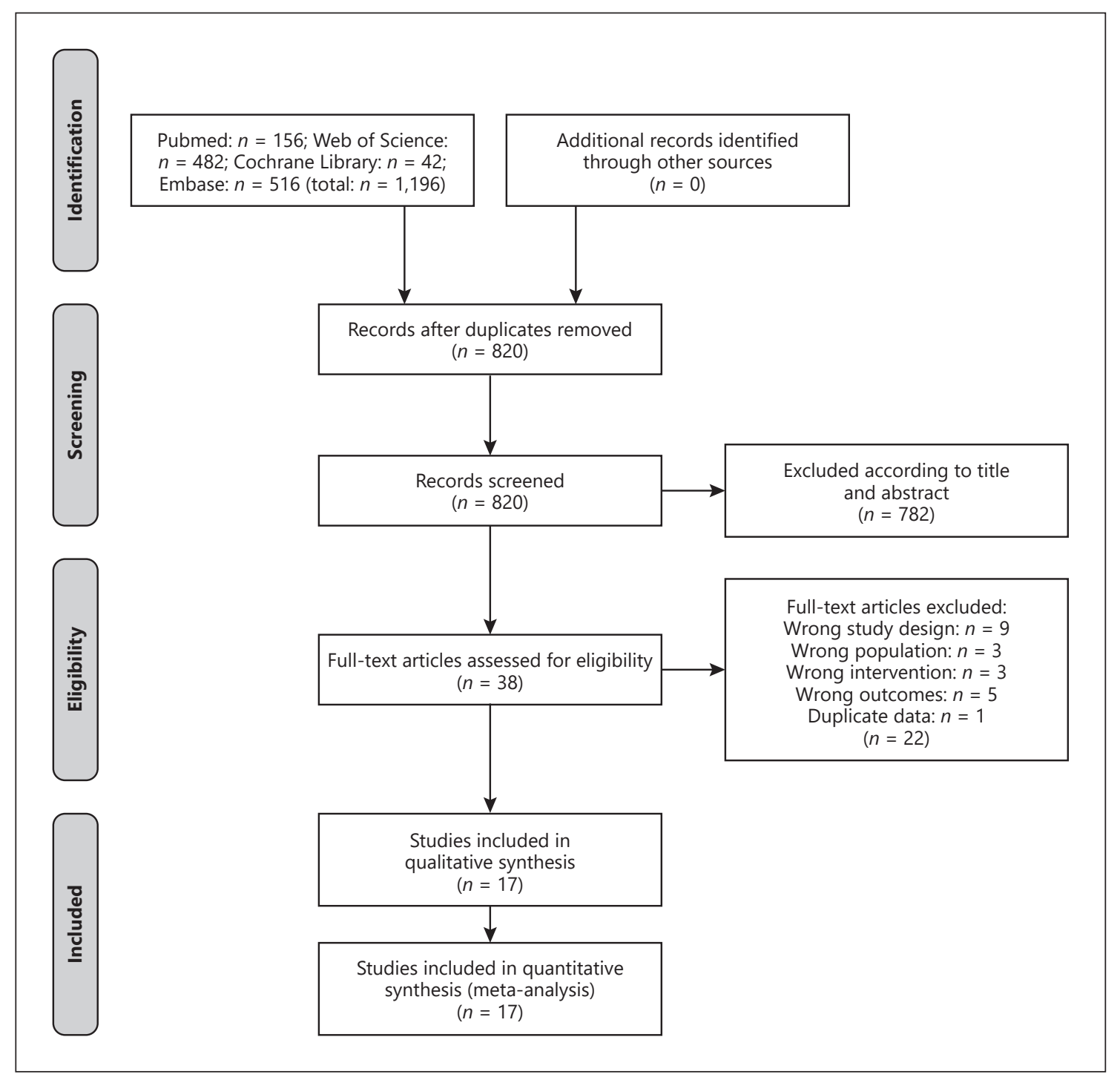

Fig. 1. Flowchart showing the selection of studies for the meta-analysis.

concealment, the blinding method, withdrawal, and loss of access [19]. NOS scores were primarily used to assess the representativeness, comparability, and follow-up integrity of exposed and nonexposed cohorts [20]. In this study, the Jadad score is considered to be greater than or equal to 4 points and the NOS score is considered to be greater than or equal to 7 points for high-quality literature.

\section{Statistical Analysis}

Cochrane Review Manager 5.3 (China) and Stata software were used for all statistical and meta-analyses, and $p<0.05$ was considered statistically significant. The effect size of continuous variables was estimated by calculating the mean difference (MD) of the results and their $95 \% \mathrm{CI}$, and the combined effect was estimated. The effect size of binary variables was estimated by calculating the OR of the results and their 95\% CI, and the combined effect was estimated. Heterogeneity was assessed using inconsistency $\left(I^{2}\right)$ statis- tics. We believe that if $I^{2}$ is greater than $50 \%$ then the heterogeneity is considerable and the random-effects model should be adopted. When $I^{2}$ is less than $50 \%$, the heterogeneity is within an acceptable range and the fixed-effects model should be adopted.

\section{Sensitivity Analysis and Publication Bias}

Sensitivity analysis was performed using Stata software to identify the source of heterogeneity. Heterogeneity and pooled effect values were recalculated after each included study was eliminated in turn. We determined the most likely source of heterogeneity by using the forest maps provided by Stata software that sequentially excluded each study. After finding the source of heterogeneity, we analyzed the experiment design, sample size, outcome evaluation criteria, and other aspects to explain the reasons for the emergence of heterogeneity and judged the reliability of statistical results.

Publication bias was quantified by the Egger method. When the $p$ value obtained by the Egger method was $>0.05$, there was no 
Table 1. Literature information and quality evaluation results

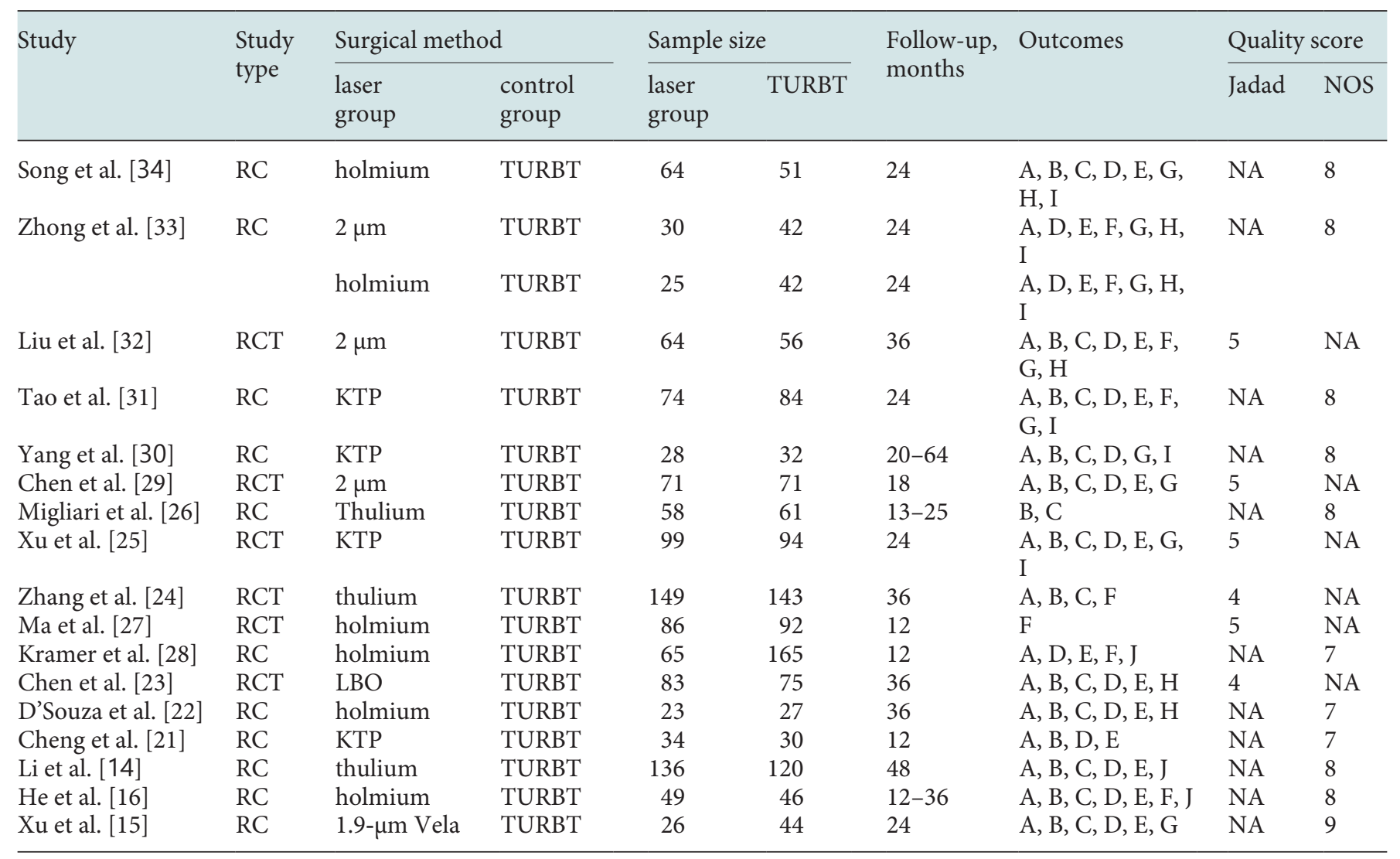

A, operation time; B, obturator nerve reflex; C, bladder perforation; D, catheterization time; E, hospitalization time; F, 12-month recurrence rate; G, 24-month recurrence rate; $\mathrm{H}$, urethral stricture; I, bladder irrigation; J, detrusor muscle acquisition; RC, retrospective cohort; KTP, potassium-titanyl-phosphate; NA, not available.

significant publication bias. On the contrary, it indicated the existence of a publication bias. If a publication bias existed, we tested the effect of publication bias on the results by pruning and filling. If a publication bias was found to have a significant impact on the results, it is discussed in detail in the Discussion.

\section{Results}

\section{Literature Retrieval Results and Basic Characteristics}

We consulted a large amount of literature and conducted careful research and screening; the specific process is shown in Figure 1. The results showed a total of 1,196 relevant studies chiefly from electronic databases. After removing duplicates, 782 references were excluded based on the title and abstract because they were reviews or letters or irrelevant to the subject of this study. By reading the full text of 38 cations, 17 studies were eventually included [14-16, 21-34], comprising 2,439 participants. Of the 17 studies, 6 were RCT [23-25, 27, $29,32]$ and the rest were retrospective cohort studies $[14-16,21,22,26,28,30,31,33,34]$. The included studies ranged from 2010 to 2018 , with a median follow-up of 24 months (range: 12-64 months). In our meta-analysis, the main characteristics and data of each study are shown in Table 1.

\section{Methodological Quality Assessment}

We used the Jadad score [19] to evaluate the RCT and the NOS [20] to evaluate the retrospective cohort studies. Through detailed evaluation, we found that the Jadad scores of all RCT were greater than or equal to 4 , and the NOS scores of all of the retrospective cohorts were greater than or equal to 7 , suggesting that all of the included studies were of good quality. The scores for each study are shown in Table 1. 


\section{Meta-Analysis Results}

Operation Time

Fifteen studies [14-16, 21-25, 28-34] reported the difference in operating time between transurethral laser surgery and TURBT therapy for NMIBC. The overall heterogeneity was considerable $\left(I^{2}=82 \%\right)$, and meta-analysis results using the random-effects model $(\mathrm{MD}=-0.2 ; 95 \%$ CI -2.29 to $1.89 ; p=0.85$ ) showed no statistical significance (Fig. 2a).

\section{Obturator Nerve Reflex}

A total of 14 studies [14-16, 21-26, 29-32, 34], involving 1,847 patients, showed that the incidence of obturator nerve reflex during transurethral laser surgery was significantly lower than with TURBT through a fixed-effects model meta-analysis $(\mathrm{OR}=0.04$; 95\% CI $0.02-0.09$; $p<$ $0.00001) . I^{2}=0 \%$ meant that no significant heterogeneity was found (Fig. 2b).

\section{Bladder Perforation}

A total of 12 studies [14-16, 22-25, 29-32,34] reported the rate of bladder perforation, and the fixed-effects model meta-analysis showed that the risk of bladder perforation in transurethral laser surgery was only 0.09 times that of TURBT (OR $=0.09 ; 95 \%$ CI $0.04-0.23$; $p<0.00001)$. $I^{2}=0$ meant that no significant heterogeneity was found (Fig. 2c).

\section{Detrusor Muscle Acquisition}

A total of 3 studies $[14,16,28]$ reported the rate of bladder perforation, and meta-analysis of the fixed-effects model showed that the detrusor muscle acquisition rate in transurethral laser surgery was 5.28 times higher than that of TURBT $(\mathrm{OR}=5.28$; 95\% CI 2.42-11.49; $p<$ $0.0001) \cdot I^{2}=0$ meant that no significant heterogeneity was found (Fig. 2d).

\section{Catheterization Time and Hospitalization Time}

A total of 15 studies [14-16, 21-23, 25, 26, 28-34] reported the postoperative urinary catheter retention time, and 13 reported the postoperative hospitalization time $[14-16,21-23,25,28,29,31-34]$. Meta-analysis of the random-effects model showed that the postoperative catheterization time $(\mathrm{MD}=-1.05 ; 95 \% \mathrm{CI}-1.41$ to -0.68 ; $p<0.00001$ ) (Fig. 2e) and hospitalization time (MD = -0.96 ; $95 \%$ CI -1.59 to -0.33 ; $p=0.003$ ) (Fig. 2f) after transurethral laser surgery were significantly shorter than with TURBT. $I^{2}$ in both cases was $97 \%$, indicating significant heterogeneity.
Bladder Irrigation and Urethral Stricture

A meta-analysis of 5 studies [22, 29, 32-34] using fixed-effects models found no significant difference in postoperative urethral stricture rates between transurethral laser surgery and TURBT $(\mathrm{OR}=0.7$; 95\% CI $0.24-$ $2.06 ; p=0.52$ ) (Fig. 2g). Five studies [22, 29, 32-34] were meta-analyzed using fixed-effects models and found that the probability of needing bladder irrigation after transurethral laser surgery was significantly lower than with TURBT $(\mathrm{OR}=0.21 ; 95 \%$ CI $0.13-0.35 ; p<0.00001)$ (Fig. 2h). $I^{2}$ was 0 and $9 \%$, respectively.

\section{Postoperative Recurrence Rate}

Recurrence rates at 12 months were reported in 7 articles $[16,24,27,28,31-33]$ and recurrence rates at 24 months were reported in 8 articles [15, 25, 29-34]. A meta-analysis of fixed-effect models showed that the recurrence rates of transurethral laser surgery at 12 months $(\mathrm{OR}=0.66$; 95\% CI $0.48-0.9 ; p=0.008)$ (Fig. $2 \mathrm{i})$ and 24 months $(\mathrm{OR}=0.6$; 95\% CI 0.41-0.86; $p=0.005)$ (Fig. 2j) were significantly lower than with TURBT. $I^{2}=0$ meant that no significant heterogeneity was found.

\section{Subgroup Analysis}

Our study included 17 articles comprising 6 RCTS and 11 retrospective cohort studies. In order to investigate whether the study type would have a significant bias on our results, we conducted a subgroup analysis based on the study type, and the results showed that there was no significant difference in meta-analysis results between the RCT subgroup and the retrospective cohort subgroup. It is worth noting that the meta-analysis results of the 12 -month recurrence rate were inversely correlated with the overall results in both subgroups, showing no statistical difference. This study mainly includes several kinds of laser, such as holmium laser, KTP, 2-micron laser, and thulium laser. In order to further clarify whether there are differences among different kinds of laser, we conducted subgroup analyses according to laser types. Subgroup analyses showed that holmium lasers appeared to have superior efficacy and safety compared to several other lasers in different outcome measures. Specific subgroup results are available in Table 2.

Fig. 2. Forest plot and meta-analysis of: operation time (a), obturator nerve reflex (b), bladder perforation (c), detrusor muscle acquisition (d), catheterization time (e), hospitalization time (f), bladder irrigation $(\mathbf{g})$, urethral stricture $(\mathbf{h})$ and $12-(\mathbf{i})$ and 24-month-recurrence rates (j) [14-16, 21-26, 28-34].

(For figure see next page.) 


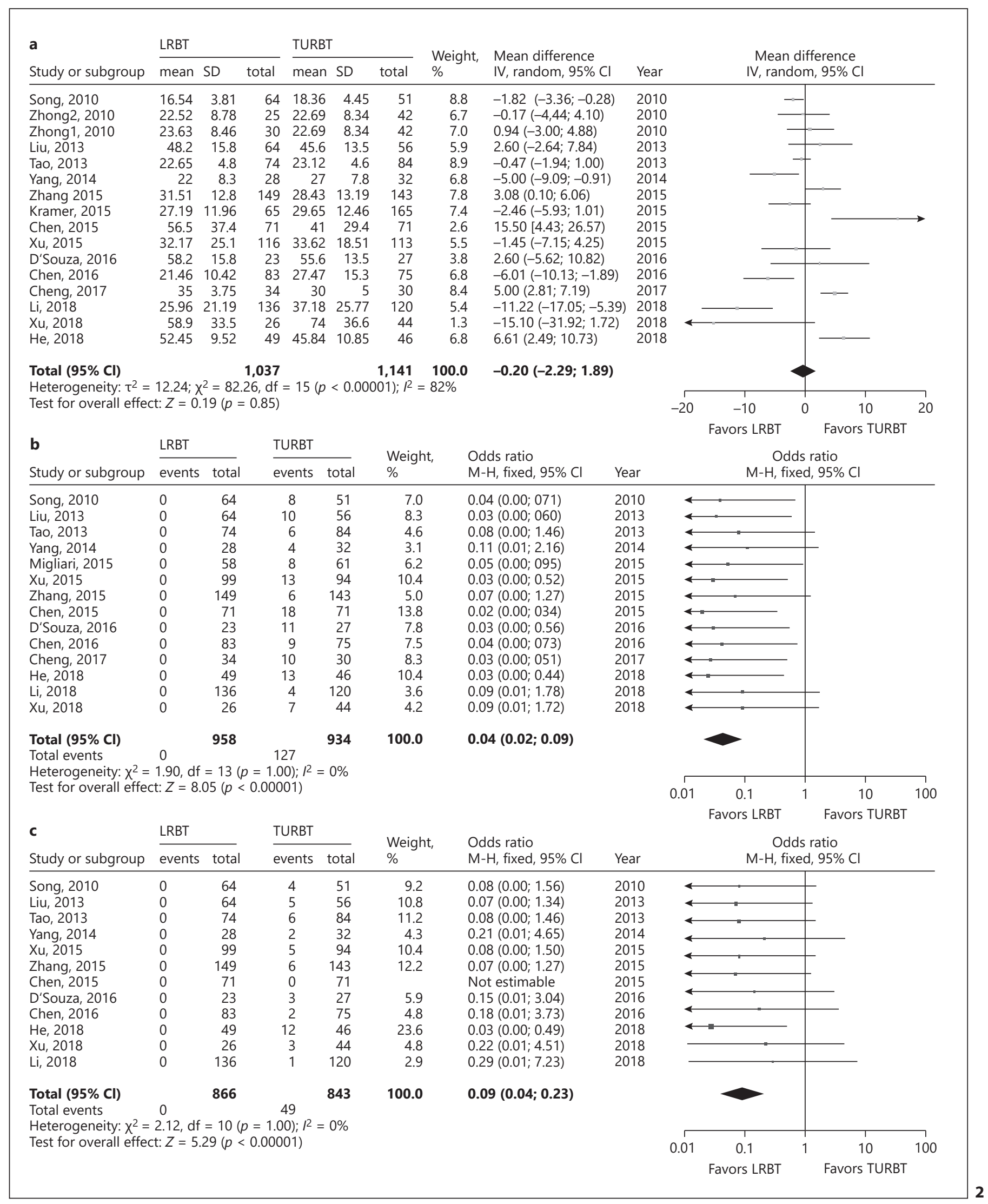

(Figure continued on next pages.)

Transurethral Laser Surgery vs.

Transurethral Resection for NMIBC
Urol Int 2020;104:810-823

DOI: $10.1159 / 000506655$ 


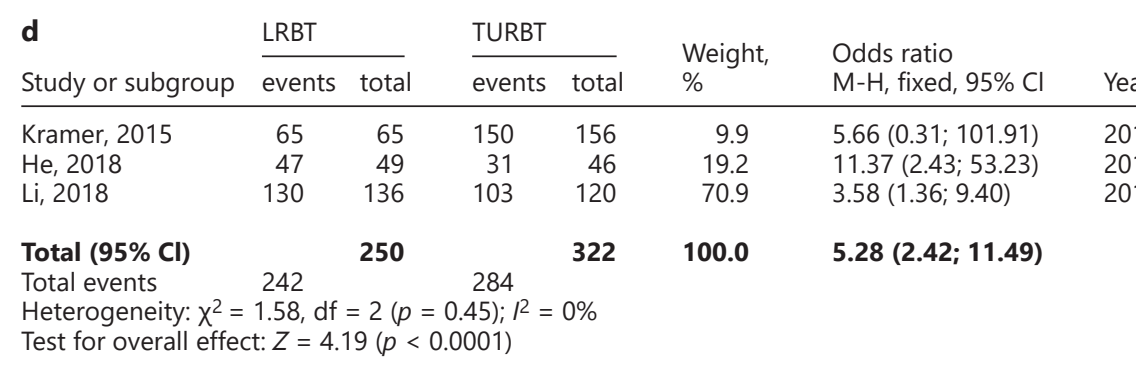

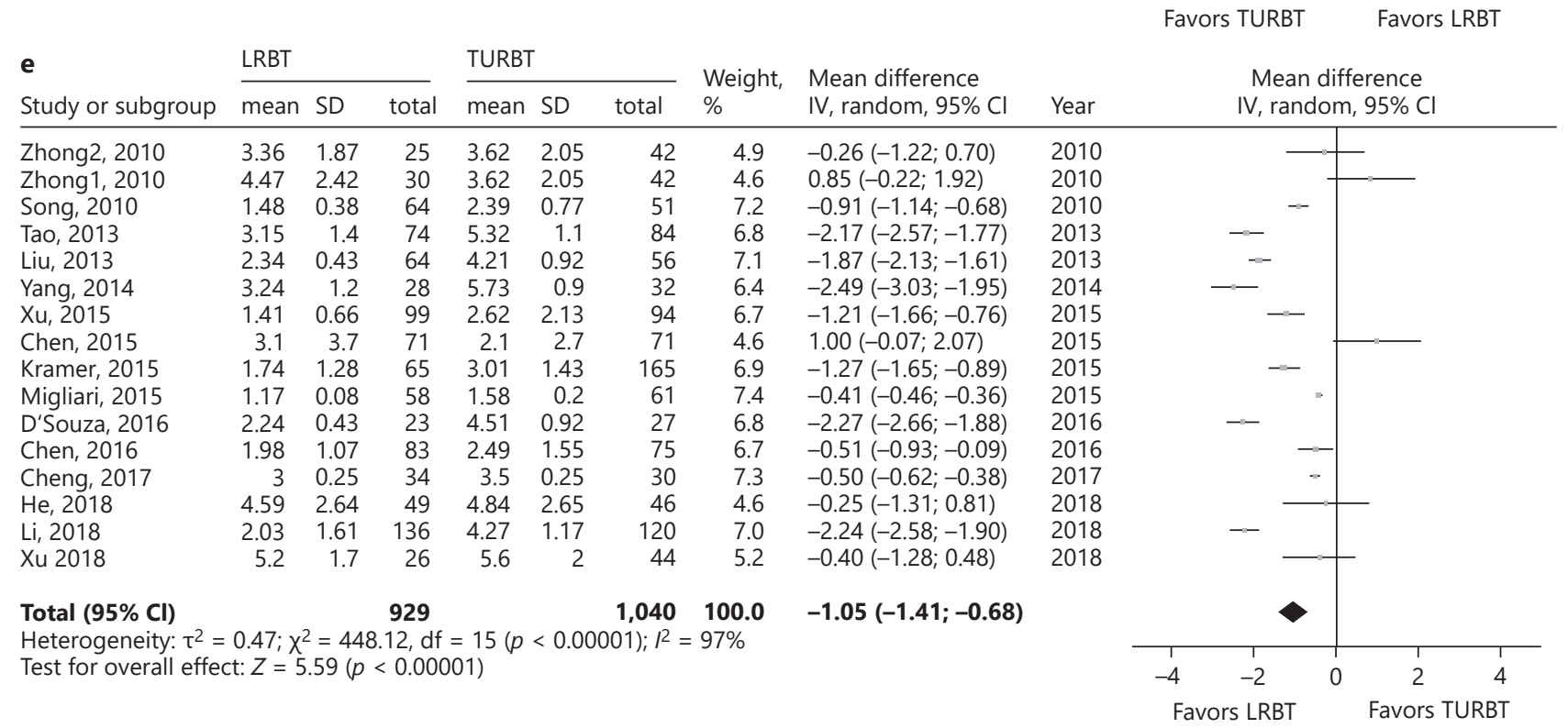

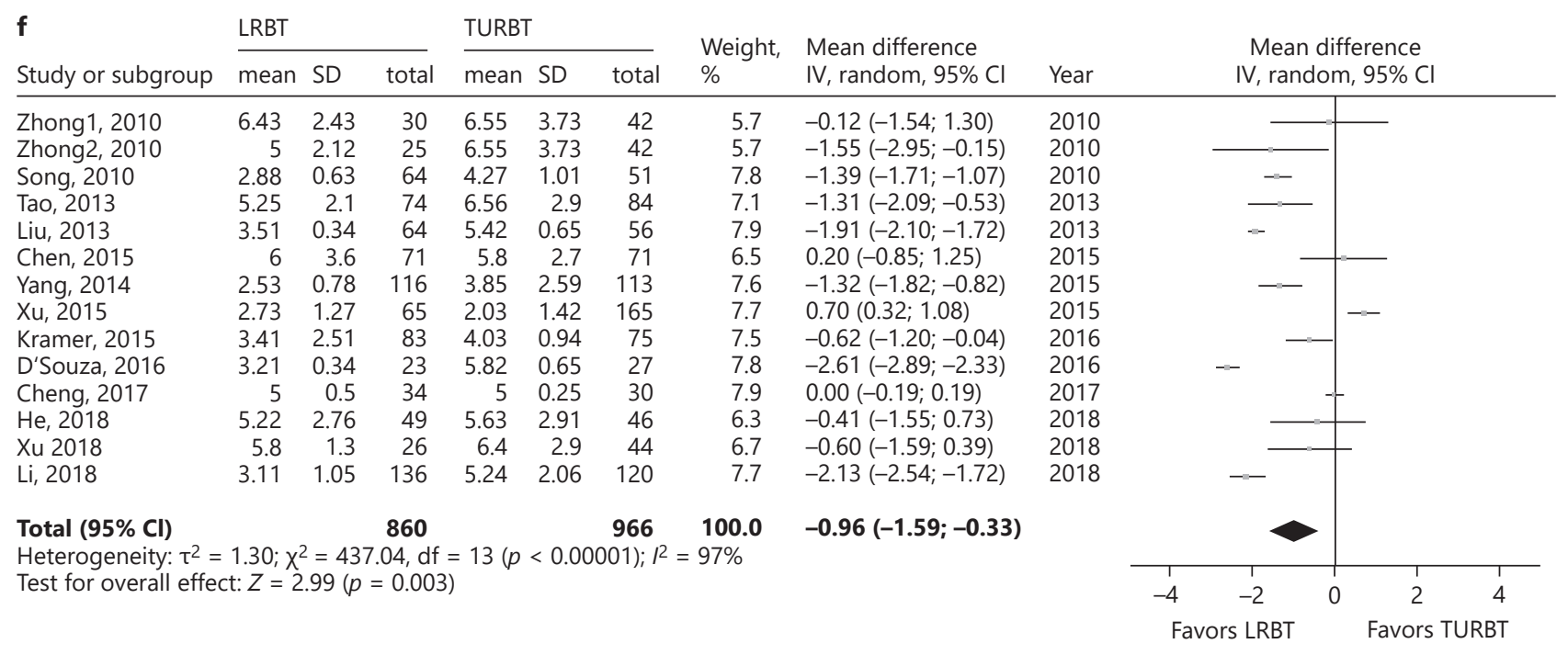




\begin{tabular}{|c|c|c|c|c|c|c|c|c|c|c|c|}
\hline \multirow{3}{*}{$\begin{array}{l}\mathbf{g} \\
\text { Study or subgroup } \\
\text { Zhong2 } 2010\end{array}$} & \multicolumn{2}{|l|}{ LRBT } & \multicolumn{2}{|l|}{ TURBT } & \multirow{2}{*}{$\begin{array}{l}\text { Weight, } \\
\%\end{array}$} & \multirow{2}{*}{$\begin{array}{l}\text { Odds ratio } \\
\mathrm{M}-\mathrm{H} \text {, fixed, } 95 \% \mathrm{Cl}\end{array}$} & \multirow[b]{2}{*}{ Year } & \multirow{2}{*}{\multicolumn{3}{|c|}{$\begin{array}{c}\text { Odds ratio } \\
\mathrm{M}-\mathrm{H} \text {, fixed, } 95 \% \mathrm{Cl}\end{array}$}} & \\
\hline & \multicolumn{2}{|c|}{ events total } & \multicolumn{2}{|c|}{ events total } & & & & & & & \\
\hline & 12 & 25 & 32 & 42 & 16.5 & $0.29(0.10 ; 0.83)$ & 2010 & & $\longrightarrow-$ & & \\
\hline Zhong1, 2010 & 19 & 30 & 32 & 42 & 13.0 & $0.54(0.19 ; 1.51)$ & 2010 & & $\longrightarrow-$ & - & \\
\hline Song, 2010 & 3 & 64 & 11 & 51 & 15.5 & $0.18(0.05 ; 0.68)$ & 2010 & & & & \\
\hline Tao, 2013 & 0 & 74 & 10 & 84 & 13.0 & $0.05(0.00 ; 0.83)$ & 2013 & 4 & & & \\
\hline Yang, 2014 & 1 & 28 & 7 & 32 & 8.4 & $0.13(0.02 ; 1.15)$ & 2014 & & & & \\
\hline $\mathrm{Xu}, 2015$ & 5 & 99 & 26 & 94 & 33.6 & $0.14(0.05 ; 0.38)$ & 2015 & & $\longrightarrow$ & & \\
\hline Total $(95 \% \mathrm{Cl})$ & & 320 & & 345 & 100.0 & $0.21(0.13 ; 0.35)$ & & & & & \\
\hline Total events & 40 & & 118 & & & & & & & & \\
\hline \multirow{2}{*}{\multicolumn{8}{|c|}{$\begin{array}{l}\text { Heterogeneity: } \chi^{2}=5.51, \mathrm{df}=5(p=0.36) ; I^{2}=9 \% \\
\text { Test for overall effect: } Z=6.12(p<0.00001)\end{array}$}} & \multirow{3}{*}{0.01} & 1 & 1 & $\neg$ \\
\hline & & & & & & & & & 0.1 & 10 & 100 \\
\hline & & & & & & & & & Favors TURBT & Favors & \\
\hline $\mathbf{h}$ & \multicolumn{2}{|l|}{ LRBT } & \multicolumn{2}{|l|}{ TURBT } & \multirow{2}{*}{$\begin{array}{l}\text { Weight, } \\
\%\end{array}$} & Oddc ratio & & & & ratis & \\
\hline Study or subgroup & events & total & events & total & & $\mathrm{M}-\mathrm{H}$, fixed, $95 \% \mathrm{Cl}$ & Year & & $\mathrm{M}-\mathrm{H}$, fixed & d, $95 \% \mathrm{Cl}$ & \\
\hline Zhong1, 2010 & 0 & 30 & 0 & 42 & & Not estimable & 2010 & & & & \\
\hline Song, 2010 & 1 & 64 & 1 & 51 & 14.5 & $0.79(0.05 ; 13.01)$ & 2010 & & & & \\
\hline Zhong2, 2010 & 0 & 25 & 0 & 42 & & Not estimable & 2010 & & & & \\
\hline Liu, 2013 & 2 & 64 & 2 & 56 & 27.3 & $0.87(0.12 ; 6.39)$ & 2013 & & & & \\
\hline Chen, 2016 & 1 & 83 & 3 & 75 & 41.2 & $0.29(0.03 ; 2.88)$ & 2016 & & & & \\
\hline D'Souza, 2016 & 2 & 23 & 3 & 75 & 17.0 & $2.29(0.36 ; 14.60)$ & 2016 & & & $\longrightarrow$ & \\
\hline Total $(95 \% \mathrm{Cl})$ & & 289 & & 341 & 100.0 & $0.86(0.30 ; 2.47)$ & & & & & \\
\hline Total events & 6 & & 9 & & & & & & & & \\
\hline Heterogeneity: $\chi^{2}=$ & 1.92, df & $=3(p$ & $.59) ; R^{2}=$ & & & & & & 1 & 1 & \\
\hline Test for overall effec & $\mathrm{t}: Z=0.2$ & $28(p=$ & & & & & & 0.01 & 0.1 & 10 & 100 \\
\hline & & & & & & & & & Favors LRBT & Favors T & \\
\hline i & LRBT & & TURBT & & & & & & & & \\
\hline Study or subgroup & events & total & events & total & $\%$ & $\mathrm{M}-\mathrm{H}$, fixed, $95 \% \mathrm{Cl}$ & Year & & $\mathrm{M}-\mathrm{H}$, fixed & d, $95 \% \mathrm{Cl}$ & \\
\hline Zhong1, 2010 & 2 & 27 & 4 & 39 & 3.0 & $0.70(0.12 ; 4.12)$ & 2010 & & $\bar{\Gamma}$ & - & \\
\hline Zhong2, 2010 & 1 & 23 & 4 & 39 & 2.8 & $0.40(0.04 ; 3.79)$ & 2010 & & & ( & \\
\hline Tao, 2013 & 1 & 74 & 4 & 84 & 3.7 & $0.27(0.03 ; 2.51)$ & 2013 & & & - & \\
\hline Liu, 2013 & 7 & 64 & 6 & 56 & 5.7 & $1.02(0.32 ; 3.25)$ & 2013 & & - & & \\
\hline Kramer, 2015 & 10 & 54 & 23 & 94 & 13.7 & $0.70(0.31 ; 1.61)$ & 2015 & & $=$ & - & \\
\hline Ma, 2015 & 16 & 86 & 35 & 92 & 27.5 & $0.37(0.19 ; 0.74)$ & 2015 & & $\longrightarrow$ & & \\
\hline Zhang, 2015 & 45 & 149 & 46 & 143 & 32.7 & $0.91(0.56 ; 1.50)$ & 2015 & & & & \\
\hline $\mathrm{He}, 2018$ & 9 & 49 & 13 & 46 & 10.9 & $0.57(0.22 ; 150)$ & 2018 & & | & - & \\
\hline Total $(95 \% \mathrm{Cl})$ & & 526 & & 593 & 100.0 & $0.66(0.48 ; 0.90)$ & & & $\bullet$ & & \\
\hline $\begin{array}{l}\text { Total events } \\
\text { Heterogeneity } \gamma^{2}=\end{array}$ & $\begin{array}{l}91 \\
578 d f\end{array}$ & $=71 \mathrm{n}$ & $\begin{array}{c}135 \\
57 \cdot 1^{2}=\end{array}$ & & & & & & & & \\
\hline Test for overall effec & $\mathrm{t}: Z=2$ & $64(p=$ & 08) & & & & & 0.01 & 0.1 & 10 & 100 \\
\hline & & & & & & & & & Favors LRBT & Favors T & \\
\hline $\mathbf{j}$ & LRBT & & TURBT & & Weiaht & Odds ratio & & & Odds & s ratio & \\
\hline Study or subgroup & events & total & events & total & $\%$ & $\mathrm{M}-\mathrm{H}$, fixed, $95 \% \mathrm{Cl}$ & Year & & $\mathrm{M}-\mathrm{H}$, fixed & d, $95 \% \mathrm{Cl}$ & \\
\hline Zhong1, 2010 & 2 & 24 & 3 & 32 & 3.1 & $0.88(0.14 ; 5.72)$ & 2010 & & & & \\
\hline Zhong2, 2010 & 1 & 20 & 3 & 32 & 2.9 & $0.51(0.05 ; 5.26)$ & 2010 & & & & \\
\hline Song, 2010 & 20 & 63 & 23 & 51 & 22.7 & $0.57(0.26 ; 1.22)$ & 2010 & & $\longrightarrow$ & & \\
\hline Liu, 2013 & 12 & 64 & 13 & 56 & 14.7 & $0.76(0.32 ; 1.84)$ & 2013 & & & - & \\
\hline Tao, 2013 & 1 & 74 & 6 & 34 & 7.2 & $0.18(0.02 ; 1.52)$ & 2013 & & & & \\
\hline Yang, 2014 & 1 & 28 & 3 & 32 & 3.5 & $0.36(0.04 ; 3.65)$ & 2014 & & 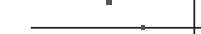 & & \\
\hline Chen, 2015 & 4 & 71 & 7 & 71 & 8.6 & $0.55(0.15 ; 1.95)$ & 2015 & & $\longrightarrow$ & E & \\
\hline Xu, 2015 & 21 & 99 & 26 & 94 & 27.4 & $0.70(0.36 ; 1.36)$ & 2015 & & $\rightarrow-$ & + & \\
\hline Xu, 2018 & 4 & 26 & 12 & 44 & 9.9 & $0.48(0.14 ; 1.70)$ & 2018 & & $\cdots$ & - & \\
\hline Total $(95 \% \mathrm{Cl})$ & & 469 & & 496 & 100.0 & $0.60(0.41 ; 0.86)$ & & & $>$ & & \\
\hline $\begin{array}{l}\text { Total events } \\
\text { Heterogeneity } \gamma^{2}=\end{array}$ & 66 & $=8 / n$ & 96 & $0 \%$ & & & & & & & \\
\hline Test for overall effec & $\mathrm{t}: Z=2$. & $79(p=$ & 05) & & & & & 0.01 & 0.1 & 10 & 100 \\
\hline & & & & & & & & & Favors LRBT & Favors T & \\
\hline
\end{tabular}

Transurethral Laser Surgery vs.

Transurethral Resection for NMIBC 
Table 2. Subgroup analysis of study type and laser type

\begin{tabular}{|c|c|c|c|c|c|c|}
\hline \multicolumn{7}{|l|}{ Study type } \\
\hline RCT & $0.05(0.02$ to 0.13$)$ & $0.09(0.02$ to 0.38$)$ & $-1.35(-1.55$ to -1.15$)$ & $0.14(0.0$ to 0.38$)$ & $0.70(0.48$ to 1.01$)$ & $\begin{array}{l}0.69(0.43 \text { to } \\
1.13)\end{array}$ \\
\hline $\mathrm{RC}$ & 0.03 (0.01 to 0.12$)$ & $0.10(0.03$ to 0.29$)$ & $-0.55(-0.60$ to -0.51$)$ & $0.25(0.14$ to 0.44$)$ & $0.59(0.34$ to 1.02$)$ & $\begin{array}{l}0.57(0.37 \text { to } \\
0.87)\end{array}$ \\
\hline \multicolumn{7}{|c|}{ Laser type } \\
\hline Holmium & $0.03(0.0$ to 0.16$)$ & $0.06(0.01$ to 0.31$)$ & $-1.21(-1.38$ to -1.04$)$ & $0.24(0.10$ to 0.54$)$ & $0.50(0.31$ to 0.78$)$ & $\begin{array}{l}0.56(0.27 \text { to } \\
1.16)\end{array}$ \\
\hline KTP & $0.04(0.01$ to 0.16$)$ & $0.10(0.02$ to 0.56$)$ & $-0.76(-0.87$ to -0.65$)$ & $0.12(0.05$ to 0.28$)$ & 0.27 (0.03 to 2.51$)$ & $\begin{array}{l}0.57(0.32 \text { to } \\
1.04)\end{array}$ \\
\hline $2 \mu \mathrm{m}$ & $0.03(0.00$ to 0.19$)$ & 0.07 (0.00 to 1.34$)$ & $-0.05(-2.270$ to 2.16$)$ & $0.54(0.19$ to 1.51$)$ & $0.91(0.35$ to 2.38$)$ & $\begin{array}{l}0.71(0.36 \text { to } \\
1.39)\end{array}$ \\
\hline
\end{tabular}

RC, retrospective cohort. Values are presented as MD (95\% CI).

\section{Sensitivity Analysis}

There was significant heterogeneity in the analysis of operation time, catheterization time, and hospitalization time $(82,97$, and $97 \%$, respectively). We used Stata software for sensitivity analysis and found that the results obtained after removing each study in turn were between the 2 bounds (Fig. 3). At the same time, after removing each study in turn, the $I^{2}$ of the corresponding outcome indicators did not change significantly. In the operation time group, the metaanalysis results changed from $\mathrm{MD}=-0.2(95 \% \mathrm{CI}-2.29$ to $1.89 ; p=0.85)$ to $\mathrm{MD}=-0.11(95 \% \mathrm{CI}-0.87$ to $0.66 ; p=$ $0.79)$ after we switched the random-effects model to the fixed-effects model. In the outcome index group of catheterization time, the results of the meta-analysis changed from $\mathrm{MD}=-1.05(95 \% \mathrm{CI}-1.41$ to $-0.68 ; p<0.00001)$ to $\mathrm{MD}=-0.6(95 \% \mathrm{CI}-0.64$ to $-0.55 ; p<0.00001)$. In the outcome index group of hospitalization time, the results of the meta-analysis changed from $\mathrm{MD}=-0.96(95 \% \mathrm{CI}-1.59$ to $-0.33 ; p=0.003)$ to $\mathrm{MD}=-1.15(95 \% \mathrm{CI}-1.25$ to -1.05 ; $p<0.00001)$. The meta-analysis results of the 3 outcome indicator groups did not change after switching to the fixedeffects model. At the same time, combined with the forest map of sensitivity analysis obtained by Stata software, we found that although the 3 outcome indicator groups all had obvious heterogeneity they had no significant impact on the results, and the results we obtained are stable and credible.

\section{Publication Bias}

Publication bias was quantitatively analyzed by the Egger method. Publication bias was found in the 5 outcome indicator groups including catheterization time, obturator nerve reflex, bladder perforation, bladder perfusion, and detrusor muscle acquisition rate $(p<0.05)$ (Fig. 4). Further verification by the trim-and-fill method showed that, although publication bias may exist, our results were robust [35].

\section{Discussion}

TURBT, as the standard surgical treatment for NMIBC, has the advantages of less trauma, a faster postoperative recovery, and maintenance of the quality of life of the patient by retention of the bladder [36]. However, the commonly used transurethral resection has complications such as obturator nerve reflex and bladder perforation [5]. At the same time, as a process of "incise and scatter," the spread of bladder tumor cells and incomplete tumor resection also limit its application [37]. In addition, TURBT is not suitable for patients with anticoagulants or patients with artificial pacemakers because the anticoagulant increases the risk of bleeding and the flow of electricity in the tissues during TURBT has the potential to interfere with the pacemaker $[38,39]$. Laser application is a new, safe, and efficient technology, especially in urology. Several studies have reported the advantages of transurethral laser surgery in the treatment of NMIBC, including a low incidence of intraoperative obturator nerve reflex and bladder perforation, a rapid postoperative recovery, complete pathological specimens, and a low recurrence 
Fig. 3. Forest plots for sensitivity analysis [14-16, 21-26, 28-34].

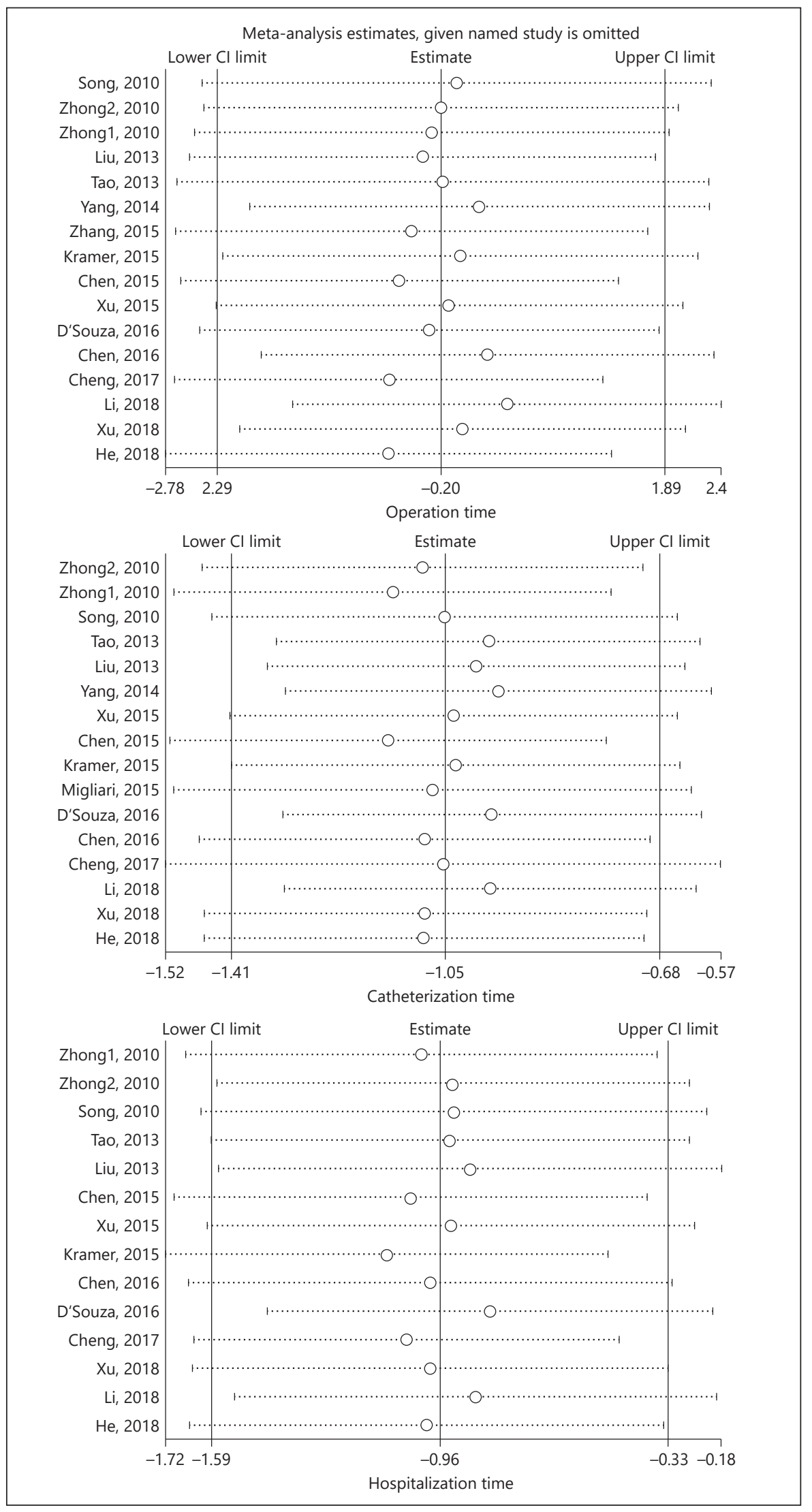



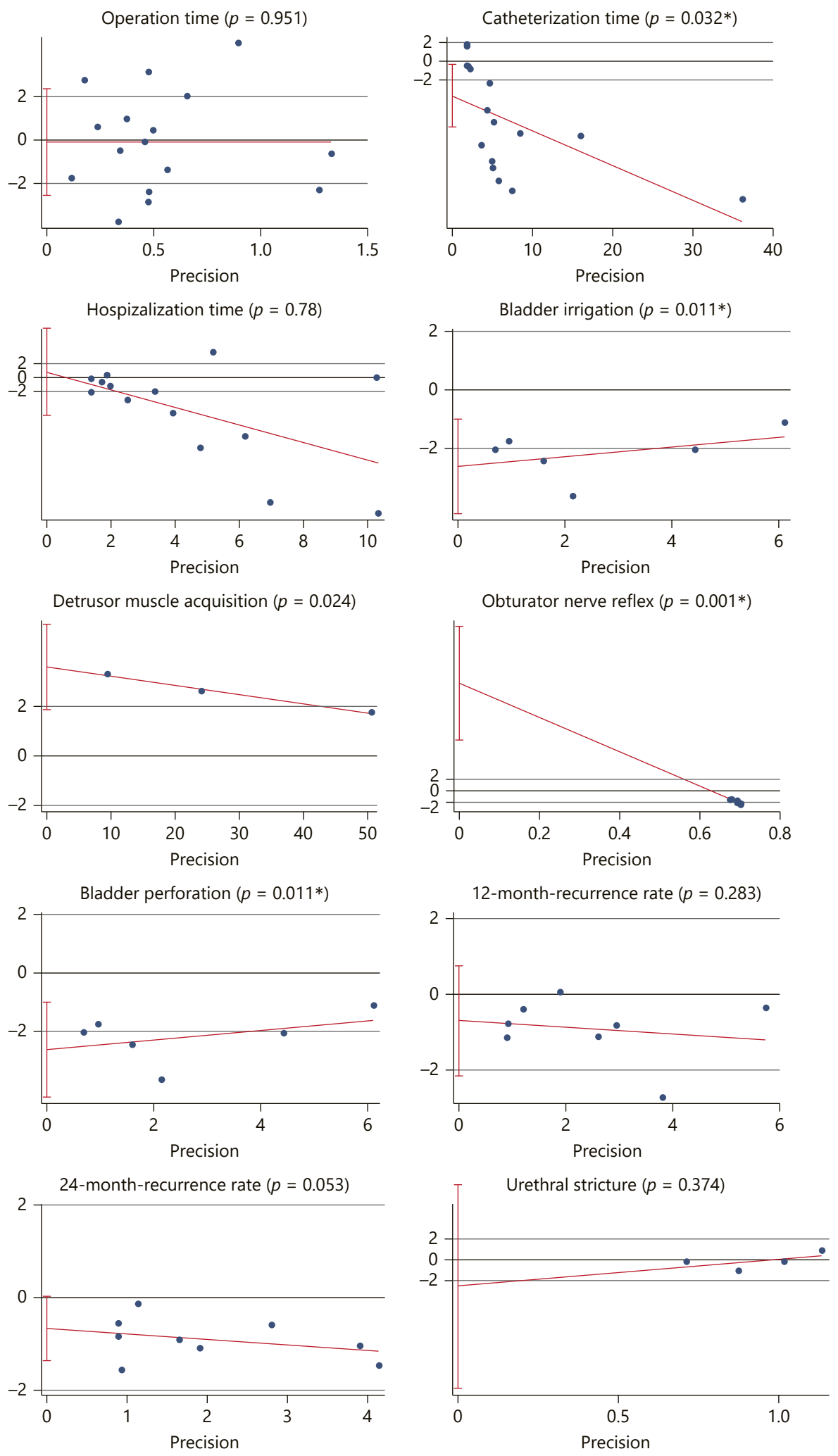

Fig. 4. Publication bias (Egger graphs). 
rate [14-16, 21-23]. Studies have also demonstrated that it can be selectively absorbed by hemoglobin and does not use an electric current, so it has a higher safety for patients using anticoagulants and patients with artificial pacemakers, which greatly expands the applicable patient population of transurethral laser surgery [22, 28]. Judging from the above research results, transurethral laser surgery compared to TURBT has great advantages, but the sample size of these studies is small and proved insufficient; there is still a lack of large-sample RCT, so we conducted the meta-analysis included 2,439 participants in order to draw strong conclusions and provide a strong basis for clinical decision making.

As far as we know, our meta-analysis is the latest and most comprehensive. Ours is the first meta-analysis to analyze the recurrence rate at 12 months and the first to conduct subgroup analysis based on research type and laser type. The results of our meta-analysis showed no statistically significant difference in operative time or postoperative incidence of urethral stricture for transurethral laser surgery showed compared to transurethral resection of bladder tumors. However, compared with TURBT, transurethral laser surgery has a lower incidence of intraoperative obturator nerve reflex and bladder perforation, a higher rate of detrusor muscle acquisition, shorter catheterization and hospitalization times, a lower probability of postoperative bladder irrigation, and lower recurrence rates at 12 and 24 months.

The reason for this result may be that during the treatment of NMIBC laser surgery and TURBT used completely different energy forms and completely different tumor removal methods. However, the basic operation techniques of transurethral surgery are similar, including endoscopic insertion through an endoscopic sheath and then insertion of the optical fiber or electric cutting ring and operation by means of swing, rotation, advance and retreat, etc. Therefore, there is no statistical difference in terms of the operation time and incidence of urethral stricture. In transurethral laser surgery, tissue is cut by laser energy. Since the surgical instrument itself is in a noncontact state with the bladder tissue and no electric current passes through the bladder tissue during the operation, no stimulation of the bladder wall nerve will be formed, thus basically eliminating the risk of obturator nerve reflex [40]. Due to the absence of current interference, transurethral laser surgery is also relatively safe for patients with pacemakers $[28,41]$. The energy of a laser at a specific wavelength is not absorbed by water during propagation but can be selectively absorbed by hemoglobin to achieve the effect of coagulation and blockage of blood vessels, thus having

Transurethral Laser Surgery vs.

Transurethral Resection for NMIBC a good hemostatic effect [30, 42]. This property reduces the probability of bladder irrigation after surgery and the duration of catheterization, and it provides a safe surgical opportunity for patients undergoing long-term anticoagulant therapy [43]. Because there is less bleeding during transurethral laser surgery, surgeons can obtain a clearer surgical field and more accurate positioning. Laser has the characteristics of high efficient tissue vaporization and good tissue solidification, and the penetration of the heat inside the tissue is shallow, so the adjacent tissue is less damaged $[28,44]$. Combined with the low incidence of obturator nerve reflex, the incidence of bladder perforation was greatly reduced. To sum up, laser surgery has a lower incidence of intraoperative and postoperative complications, and some special patients do not need a longer time for preoperative preparation, so transurethral laser surgery has a shorter hospitalization time.

Due to the precise tissue cutting capability of the laser and the superior intraoperative safety, en bloc resection of NMIBC is possible. Complete resection of the tumor, lamina propria, and detrusor can reduce the residual tumor and the dissemination of tumor cells on the one hand and provide high-quality pathological specimens for further pathological diagnosis and staging on the other hand [45]. In addition, combined with the good vaporization and coagulation effect of laser, capillaries and lymphatic vessels are effectively closed, and the possibility of tumor cell dissemination is reduced $[41,45]$. Accurate pathological staging based on high-quality specimens can also better guide the selection of further treatment plans. Based on the above characteristics, the tumor recurrence rate of the transurethral laser surgery group at 12 and 24 months was lower than that of the TURBT group.

According to the results of the subgroup analysis, we basically excluded the bias caused by research type. Statistical results of 12-month recurrence rates in both subgroups revealed no statistical significance, which may be because the sample size was too small to obtain accurate results. We need more relevant studies to further clarify the difference between transurethral laser surgery and TURBT in the future. When we did a subgroup analysis by laser type, holmium laser seemed to have better efficacy and safety. This may be related to the earliest use, widest application, and most mature technology of holmium laser in urology. Due to the limited amount of literature and the sample size, the results need to be further verified by head-to-head clinical control studies.

In our sensitivity analysis, we found no significant source of heterogeneity, but we found that our results were robust despite the presence of heterogeneity. As the 
outcome indicators of heterogeneity are all related to the statistics of time, we consider that the differences in the indicators of perioperative time may have been caused by the different surgical concepts and perioperative management concepts of different hospitals.

\section{Limitations}

It is undeniable that this study also has several limitations. First, despite the statistics on recurrence rates at 12 and 24 months, the literature volume is still small and there is a lack of long-term data. Second, due to the small amount of literature, no more-detailed subgroup analysis was performed based on laser type and TURBT type. Third, heterogeneity and publication bias may exist.

\section{Conclusion}

Transurethral laser surgery for NMIBC, as compared with TURBT, is associated with a lower incidence of intraoperative complications, a lower postoperative recurrence rate, and a faster postoperative recovery.

\section{Acknowledgement}

Special thanks go to Dr. Jianglei Zhang for his guidance on some statistical methods.

\section{Disclosure Statement}

The authors have no conflict of interests to declare.

\section{Funding Sources}

This study was funded by the science and technology program of Suzhou city (grant No. SLJ201906).

\section{Author Contributions}

Jiangnan Xu: study design, data collection, statistical analysis, and writing and submission of this paper. Chao Wang: study design, data collection, statistical analysis, revision of this paper. Jun Ouyang: study design, writing and overall planning of this paper. JiaLe Sun: data collection and partial statistical analysis. Can $\mathrm{Hu}$ : data collection.

\section{References}

1 Siegel RL, Miller KD, Jemal A. Cancer statistics, 2019. CA Cancer J Clin. 2019 Jan;69(1): 7-34.

2 Kamat AM, Hahn NM, Efstathiou JA, Lerner SP, Malmström PU, Choi W, et al. Bladder cancer. Lancet. 2016 Dec;388(10061):2796810

3 Babjuk M, Böhle A, Burger M, Capoun O, Cohen D, Compérat EM, et al. EAU Guidelines on Non-Muscle-invasive Urothelial Carcinoma of the Bladder: Update 2016. Eur Urol. 2017 Mar;71(3):447-61.

4 Nishiyama H. Asia Consensus Statement on NCCN Clinical Practice Guideline for bladder cancer. Jpn J Clin Oncol. 2018 Jan;48(1):3-6.

5 Gregg JR, McCormick B, Wang L, Cohen P, Sun D, Penson DF, et al. Short term complications from transurethral resection of bladder tumor. Can J Urol. 2016 Apr;23(2):8198-203.

6 Shah NF, Sofi KP, Nengroo SH. Obturator Nerve Block in Transurethral Resection of Bladder Tumor: A Comparison of Ultrasound-guided Technique versus Ultrasound with Nerve Stimulation Technique. Anesth Essays Res. 2017 Apr-Jun;11(2):411-5.

7 Guo CC, Al-Ahmadie HA, Flaig TW, Kamat AM. Contribution of bladder cancer pathology assessment in planning clinical trials. UrolOncol.2018Feb;S1078-1439(18)30001-2.

8 Koie T, Ohyama C, Hosogoe S, Yamamoto H, Imai A, Hatakeyama S, et al. Oncological outcomes of a single but extensive transurethral resection followed by appropriate intra-vesi- cal instillation therapy for newly diagnosed non-muscle-invasive bladder cancer. Int Urol Nephrol. 2015 Sep;47(9):1509-14.

9 Dalbagni G. Editorial comment on: detrusor muscle in the first, apparently complete transurethral resection of bladder tumour specimen is a surrogate marker of resection quality, predicts risk of early recurrence, and is dependent on operator experience. Eur Urol. 2010 May;57(5):849.

10 Korn SM, Hübner NA, Seitz C, Shariat SF, Fajkovic H. Role of lasers in urology. Photochem Photobiol Sci. 2019 Feb;18(2):295-303.

11 Xin Z, Hanmin C, Kunlin X, Xiaofeng Z, Rihai $\mathrm{X}$, Guoxi Z. The effective observation of the non muscleinvasive bladder cancer with the transurethral thulium laser radical resection of bladder tumor. J Endourol. 2019;33:A25.

12 Zhang J, Wang L, Mao S, Liu M, Zhang W, Zhang Z, et al. Transurethral en bloc resection with bipolar button electrode for non-muscle invasive bladder cancer. Int Urol Nephrol. 2018 Apr;50(4):619-23.

13 Enikeev D, Shariat SF, Taratkin M, Glybochko $\mathrm{P}$. The changing role of lasers in urologic surgery. Curr Opin Urol. 2020 Jan;30(1):24-9.

14 Li K, Xu Y, Tan M, Xia S, Xu Z, Xu D. A retrospective comparison of thulium laser en bloc resection of bladder tumor and plasmakinetic transurethral resection of bladder tumor in primary non-muscle invasive bladder cancer. Lasers Med Sci. 2019 Feb;34(1): 85-92.
$15 \mathrm{Xu} \mathrm{H}$, Ma J, Chen Z, Yang J, Yuan H, Wang $\mathrm{T}$, et al. Safety and efficacy of en bloc transurethral resection with $1.9 \mu \mathrm{m}$ Vela laser for treatment of non-muscle-invasive bladder cancer. Urology. 2018 Mar;113:246-50.

$16 \mathrm{He} \mathrm{L}, \mathrm{He} \mathrm{H}$, Li L, Chen C, Wang X, Hu Y. Efficacy comparison of holmium laser en bloc transurethral resection and transurethral resection in treatment of nonmuscle-invasive bladder cancer. Shiyong Zhongliu Zazhi. 2018;33(6):548-52.

17 Moher D, Liberati A, Tetzlaff J, Altman DG; PRISMA Group. Preferred reporting items for systematic reviews and meta-analyses: the PRISMA statement. Int J Surg. 2010;8(5): 336-41.

18 Hozo SP, Djulbegovic B, Hozo I. Estimating the mean and variance from the median, range, and the size of a sample. BMC Med Res Methodol. 2005 Apr;5(1):13.

19 Berger VW. Is the Jadad score the proper evaluation of trials? J Rheumatol. 2006 Aug;33(8): 1710-1.

20 Cook DA, Reed DA. Appraising the quality of medical education research methods: the Medical Education Research Study Quality Instrument and the Newcastle-Ottawa Scale-Education. Acad Med. 2015 Aug;90(8):1067-76.

21 Cheng B, Qiu X, Li H, Yang G. The safety and efficacy of front-firing green-light laser endoscopic en bloc photoselective vapo-enucleation of non-muscle-invasive bladder cancer. Ther Clin Risk Manag. 2017 Aug;13:983-8. 
22 D'Souza N, Verma A. Holmium laser transurethral resection of bladder tumor: our experience. Urol Ann. 2016 Oct-Dec;8(4):439-43.

23 Chen J, Zhao Y, Wang S, Jin X, Sun P, Zhang $\mathrm{L}$, et al. Green-light laser en bloc resection for primary non-muscle-invasive bladder tumor versus transurethral electroresection: A prospective, nonrandomized two-center trial with 36-month follow-up. Lasers Surg Med. 2016 Nov;48(9):859-65.

24 Zhang XR, Feng C, Zhu WD, Si JM, Gu BJ, Guo H, et al. Two Micrometer ContinuousWave Thulium Laser Treating Primary NonMuscle-Invasive Bladder Cancer: Is It Feasible? A Randomized Prospective Study. Photomed Laser Surg. 2015 Oct;33(10):517-23.

$25 \mathrm{Xu} \mathrm{Y,} \mathrm{Guan} \mathrm{W,} \mathrm{Chen} \mathrm{W,} \mathrm{Xie} \mathrm{C,} \mathrm{Ouyang} \mathrm{Y,}$ $\mathrm{Wu} \mathrm{Y}$, et al. Comparing the treatment outcomes of potassium-titanyl-phosphate laser vaporization and transurethral electroresection for primary nonmuscle-invasive bladder cancer: A prospective, randomized study. Lasers Surg Med. 2015 Apr;47(4):306-11.

26 Migliari R, Buffardi A, Ghabin H. Thulium laser endoscopic en bloc enucleation of nonmuscle-invasive bladder cancer. J Endourol. 2015 Nov;29(11):1258-62.

27 Ma T, Wang W, Jiang Z, Shao G, Guo L, Li J, et al. Narrow band imaging-assisted holmium laser resection reduced the recurrence rate of non-muscle invasive bladder cancer: a prospective, randomized controlled study. Zhonghua Yi Xue Za Zhi. 2015 Oct;95(37):3032-5.

28 Kramer MW, Rassweiler JJ, Klein J, Martov A, Baykov N, Lusuardi L, et al. En bloc resection of urothelium carcinoma of the bladder (EBRUC): a European multicenter study to compare safety, efficacy, and outcome of laser and electrical en bloc transurethral resection of bladder tumor. World J Urol. 2015 Dec; 33(12):1937-43.

29 Chen X, Liao J, Chen L, Qiu S, Mo C, Mao X, et al. En bloc transurethral resection with 2-micron continuous-wave laser for primary non-muscle-invasive bladder cancer: a randomized controlled trial. World J Urol. 2015; 33(7):997.
30 Yang D, Xue B, Zang Y, Liu X, Zhu J, Zhou Y, et al. Efficacy and safety of potassium-titanylphosphate laser vaporization for clinically non-muscle invasive bladder cancer. Urol J. 2014 Mar;11(1):1258-63.

31 Tao W, Yang D, Shan Y, Xue B, Sun C, Zang Y, et al. Safety and efficacy of $120 \mathrm{~W}$ high performance system greenlight laser vaporization for non-muscle-invasive bladder cancer. J XRay Sci Technol. 2013;21(2):309-16.

32 Liu H, Wu J, Xue S, Zhang Q, Ruan Y, Sun X, et al. Comparison of the safety and efficacy of conventional monopolar and 2-micron laser transurethral resection in the management of multiple nonmuscle-invasive bladder cancer. J Int Med Res. 2013 Aug;41(4):984-92.

33 Zhong C, Guo S, Tang Y, Xia S. Clinical observation on 2 micron laser for non-muscleinvasive bladder tumor treatment: single-center experience. World J Urol. 2010 Apr;28(2): 157-61.

34 Song X, Yang D, Che X, Jiang T, Li Q, Guan $\mathrm{H}$, et al. Comparing the safety and efficiency of conventional monopolar, plasmakinetic, and holmium laser transurethral resection of primary non-muscle invasive bladder cancer. J Endourol. 2010 Jan;24(1):69-73.

35 Shi L, Lin L. The trim-and-fill method for publication bias: practical guidelines and recommendations based on a large database of meta-analyses. Medicine (Baltimore). 2019 Jun;98(23):e15987.

36 Sexton WJ, Wiegand LR, Correa JJ, Politis C, Dickinson SI, Kang LC. Bladder cancer: a review of non-muscle invasive disease. Cancer Contr. 2010 Oct; 17(4):256-68.

37 Mariappan P, Zachou A, Grigor KM; Edinburgh Uro-Oncology Group. Detrusor muscle in the first, apparently complete transurethral resection of bladder tumour specimen is a surrogate marker of resection quality, predicts risk of early recurrence, and is dependent on operator experience. Eur Urol. 2010 May;57(5):843-9.
38 De Nunzio C, Franco G, Cindolo L, Autorino $\mathrm{R}$, Cicione $\mathrm{A}$, Perdonà $\mathrm{S}$, et al. Transuretral resection of the bladder (TURB): analysis of complications using a modified Clavien system in an Italian real life cohort. Eur J Surg Oncol. 2014 Jan;40(1):90-5.

39 Bolat D, Gunlusoy B, Aydogdu O, Aydin ME, Dincel C. Comparing the short-term outcomes and complications of monopolar and bipolar transurethral resection of bladder tumors in patients with coronary artery disese: a prospective, randomized, controlled study. Int Braz J Urol. 2018;44(4): 717-25.

40 Wolters M, Kramer MW, Merseburger AS, Riedl M, Leitenberger A, Martov A, et al. Laser en bloc resection of bladder tumors: A European multi-center study to evaluate safety, efficacy and outcome. Eur Urol Suppl. 2015; 14(2):eV40.

41 Kramer MW, Abdelkawi IF, Wolters M, Bach T, Gross AJ, Nagele U, et al. Current evidence for transurethral en bloc resection of nonmuscle-invasive bladder cancer. Minim Invasive Ther Allied Technol. 2014 Aug;23(4): 206-13.

42 Johnson DE, Cromeens DM, Price RE. Use of the holmium:YAG laser in urology. Lasers Surg Med. 1992;12(4):353-63.

43 Naspro R, Lerner LB, Rossini R, Manica M, Woo HH, Calopedos RJ, et al. Perioperative antithrombotic therapy in patients undergoing endoscopic urologic surgery: where do we stand with current literature? Minerva Urol Nefrol. 2018 Apr;70(2):126-36.

44 Dołowy Ł, Krajewski W, Dembowski J, Zdrojowy R, Kołodziej A. The role of lasers in modern urology. Cent European J Urol. 2015; 68(2):175-82.

45 Liang H, Yang T, Wu K, He D, Fan J. En bloc resection improves the identification of muscularis mucosae in non-muscle invasive bladder cancer. World J Urol. 2019 Dec;37(12): 2677-82.
Transurethral Laser Surgery vs. Transurethral Resection for NMIBC
Urol Int 2020;104:810-823 